\section{Bivalirudin for patients with STEMI at high risk of bleeding undergoing $\mathrm{PPCl}$}

\author{
Lu Hu, Kai Guo and Zhigang Guo
}

We read the Review by Franchi et al. (Antithrombotic therapy for patients with STEMI undergoing primary PCI. Nat. Rev. Cardiol. 14, 361-379; 2017) $)^{1}$ with great interest. We wish to discuss the role of bivalirudin for patients with ST-segment elevation myocardial infarction (STEMI) who are at high risk of bleeding and undergoing primary percutaneous coronary intervention (PPCI).

Intravenous anticoagulant therapy is mandatory in PPCI and includes heparins and bivalirudin. Currently, bivalirudin has a class I (level of evidence B) recommendation in the ACC/AHA guidelines ${ }^{2}$ for patients with STEMI undergoing PPCI. Nevertheless, the clinical benefits of bivalirudin use and whether bivalirudin is the optimal regimen have been the subject of debate in patients at high risk of bleeding (criteria defined previously $\left.{ }^{3,4}\right)$, who need more careful evaluation and attention to anticoagulant management.

In the Review, bivalirudin was identified as the optimal choice compared with heparins for anticoagulation in patients with STEMI undergoing PPCI. Meanwhile, an updated meta-analysis involving six randomized trials ( $n=17,294$ patients with STEMI undergoing PPCI) definitively showed that bivalirudin compared with heparin (with or without glycoprotein IIb/IIIa inhibitors) is associated with a reduction in major bleeding (OR 0.65, 95\% CI 0.48-0.88, $P=0.006$ ), lower all-cause mortality (OR 0.81 , 95\% CI 0.67-0.98, $P=0.03)$, and lower cardiac mortality (OR 0.69, 95\% CI 0.55-0.87, $P=0.001)^{5}$. Prolonging the bivalirudin infusion at PPCI dose $(1.75 \mathrm{mg} / \mathrm{kg} / \mathrm{h})$ for $3 \mathrm{~h}$ eliminates the excess risk of acute stent thrombosis (OR 0.81, 95\% CI 0.27-2.46, $P=0.71$ ), whereas the risk of bleeding was reduced despite treatment with high-dose bivalirudin infusion (OR 0.28, 95\% CI 0.13$0.60, P=0.001)^{5}$. Whether the clinical benefits of bivalirudin are maintained in patients with STEMI at high risk of bleeding undergoing PPCI is uncertain.
In subgroup analyses of the MATRIXSTEMI trial ${ }^{6}$ involving patients at high risk of bleeding (estimated glomerular filtration rate $<60 \mathrm{ml} / \mathrm{min}$ ), bivalirudin seemed to be associated with reduced risk of co-primary composite outcomes (major adverse cardiovascular events and net adverse clinical events), but no significant difference was observed in patients aged $\geq 75$ years with STEMI undergoing PPCI. In subgroups analyses for the primary composite outcome of major adverse cardiovascular events (or death) or major bleeding in the HEAT-PPCI ${ }^{7}$ and EUROMAX ${ }^{8}$ trials, use of bivalirudin was similar to the use of heparin in patients at high risk of bleeding (age $\geq 75$ years or creatinine clearance $\leq 60 \mathrm{ml} / \mathrm{min}$ ). In addition, investigators in the NAPLES III trial ${ }^{9}$ randomly allocated 837 biomarker-negative patients at high risk of bleeding and undergoing elective or PPCI to either bivalirudin $(n=418)$ or unfractionated heparin $(n=419)$. However, the study population was not patients with STEMI, and the results suggested no significant difference in major bleeding rate between bivalirudin and unfractionated heparin (OR 0.78, 95\% CI 0.35-1.72, $P=0.54$ ). By contrast, in the ACUITY trial ${ }^{10}$, patients aged $\geq 75$ years with non-ST-segment elevation acute coronary syndrome treated with bivalirudin alone had a significantly lower rate of non-CABG major bleeding (6.1\%) compared with those treated with heparin and glycoprotein IIb/IIIa inhibitors (12.3\%). Although the available data come from subgroup analyses of trials or study populations that did not include patients with STEMI, the evidence suggests that bivalirudin should be considered in patients with STEMI who are at high risk of bleeding and undergoing PPCI.

Overall, bivalirudin compared with heparins is likely to reduce net adverse clinical events and/or major bleeding risk, although with no significant difference in major adverse cardiovascular events. On the basis of guideline recommendations and these analyses, we believe that bivalirudin is the optimal anticoagulation regimen in these patients. Meanwhile, we look forward to implementing large-scale, prospective, randomized clinical trials involving patients at high risk of bleeding to substantiate our viewpoint.

Lu Hu, Kai Guo and Zhigang Guo are at the Department of Cardiology, Huiqiao Medical Center, Nanfang Hospital, Southern Medical University, No. 1838 North Guangzhou Avenue, Baiyun District, Guangzhou 510515, Guangdong Province, China.

Correspondence to Z.G. guozhigang126@126.com doi: 10.1038/nrcardio.2017.141 Published online 14 Sep 2017

1. Franchi, F., Rollini, F. \& Angiolillo, D. J. Antithrombotic therapy for patients with STEM undergoing primary $\mathrm{PCl}$. Nat. Rev. Cardiol. 14 361-379 (2017).

2. O'Gara, P. T. et al. 2013 ACCF/AHA guideline for the management of ST-elevation myocardial infarction: a report of the American College of Cardiology Foundation/American Heart Association Task Force on Practice Guidelines. J. Am. Coll. Cardiol. 61, e78-e140 (2013).

3. Nikolsky, E. et al. Development and validation of a prognostic risk score for major bleeding in patients undergoing percutaneous coronary intervention via the femoral approach. Eur. Heart J. 28, 1936-1945 (2007).

4. Urban, P. et al. Polymer-free drug-coated coronary stents in patients at high bleeding risk. N. Engl. J. Med. 373, 2038-2047 (2015).

5. Fahrni, G. et al. Prolonged high-dose bivalirudin infusion reduces major bleeding without increasing stent thrombosis in patients undergoing primary percutaneous coronary intervention: novel insights from an updated meta-analysis. J. Am. Heart Assoc. 5 , e003515 (2016)

6. Leonardi, S. et al. Bivalirudin or unfractionated heparin in patients with acute coronary syndromes managed invasively with and without ST elevation (MATRIX): randomised controlled trial. BMJ 354, i4935 (2016).

7. Shahzad, A. et al. Unfractionated heparin versus bivalirudin in primary percutaneous coronary intervention (HEAT-PPCI): an open-label, single centre, randomised controlled trial. Lancet 384, 1849-1858 (2014).

8. Steg, P. G. et al. Bivalirudin started during emergency transport for primary PCI. N. Engl. J. Med. 369, 2207-2217 (2013).

9. Briguori, C. et al. Novel Approaches for Preventing or Limiting Events (NAPLES) III trial: randomized comparison of bivalirudin versus unfractionated heparin in patients at increased risk of bleeding undergoing transfemoral elective coronary stenting. JACC Cardiovasc. Interv. 8, 414-423 (2015)

10. Lopes, R. D. et al. Advanced age, antithrombotic strategy, and bleeding in non-ST-segment elevation acute coronary syndromes: results from the ACUITY (Acute Catheterization and Urgent Intervention Triage Strategy) trial. J. Am. Coll. Cardiol. 53, 1021-1030 (2009).

\section{Acknowledgements}

The authors are funded by the National Natural Science Foundation of China (No. 81370380), the Major Scientific Research Foundation of colleges and universities of Guangdong province, China (No. 2016KZDXM016), and the Clinical Training Project Foundation of Southern Medical University (No. LC2016PY002).

Competing interests statement

The authors declare no competing interests. 